\title{
Obesity, osteoarthritis and pain
}

\begin{abstract}
Objectives: To describe the linkage between pain, and obesity in the context of osteoarthritis. To propose a novel approach to reducing obesity associated with painful osteoarthritis.

Methods: A comprehensive literature review covering the years 2000-2014 was conducted using major data bases and key words: behavioral self-management, obesity, osteoarthritis, and pain. Previously collected data were also examined.

Results: Obesity is common among osteoarthritis sufferers. Those who are obese experience more pain and disability than those who are normal weight. A possible deterrent to weight loss in obese osteoarthritis cases is pain. A possible solution is a multidimensional self-management program utilizing cognitive behavioral strategies that addresses control of pain as well as dietary behaviors.

Conclusion: Obesity is a risk factor for heightened pain and disability among osteoarthritis sufferers. More comprehensive non pharmaceutical behavioral strategies to reduce pain and possible depression in these patients may enable more effective and sustained weight reduction or maintenance than that achieved through standard nutritional interventions alone.
\end{abstract}

Keywords: body mass index, obesity, osteoarthritis, pain, weight, weight loss
Volume 2 Issue I - 2015

\author{
Ray Marks \\ Department of Health and Behavior Studies, Columbia \\ University, USA \\ Department of Health/Physical Education \& Gerontological \\ Studies/Services, The City University of New York, USA
}

Correspondence: Ray Marks, Department of Health and Behavior Studies, Columbia University, Teachers College, Box I I4, 525 W 120th Street, New York, NY 10027, Tel 12126783445, Fax 12126788259, Email rm226@columbia.edu

Received: August 26, 2014 | Published: January 02, 2015

\section{Introduction}

Osteoarthritis, a highly common progressively disabling condition of multifactorial origin ${ }^{1}$ may be influenced negatively by factors other than aging, such as pain. In addition a variety of co morbid conditions such as obesity, which prevails at a higher rate in people with osteoarthritis than that observed in the general population, ${ }^{2}$ may be implicated in the pathogenesis of osteoarthritis. However, attempts to ameliorate this leading global cause of pain and disability, ${ }^{3}$ among those who are obese said to experience more severe pain than those of normal weight ${ }^{4}$ may be extremely challenging if only dietary recommendations or other passive strategies, such as bariatric surgery, are forthcoming. This is because pain accompanying osteoarthritis, which is often severe, not only limits the activity level of the affected individual which may already be compromised ${ }^{5}$ even though exercise is commonly advocated for weight control, overall health maintenance, and effective energy balance, but unrelenting pain can lead to depression, ${ }^{1}$ that can foster weight gain ${ }^{6}$ sleep disturbances, use of corticosteroids psychotropic medications and narcotic agents thought to adversely impact on body weight ${ }^{8-10}$ and reduce motivation for behavioural changes. At the same time, since being overweight even in the absence of pain is associated with increases in the amount of force across a weight-bearing joint, ${ }^{1}$ activity limitations, fatigue and muscle weakness ${ }^{11}$ and the development of inflammatory products, ${ }^{1}$ and all these factors alone can hasten joint destruction the importance of reducing excess weight among overweight adults with this condition cannot be underestimated, especially in the early phases of the disease process. ${ }^{12}$ Furthermore given that excess weight is strongly linked to osteoarthritis pain in a dose dependent fashion, even though the cause effect relationship between these variables may not be conclusive,${ }^{13}$ the finding that the morbidly obese patient commonly has worse pain relief after total knee joint replacement surgery ${ }^{14}$ and arthroscopy ${ }^{15}$ and patients continue to use addictive opioids to relieve their pain ${ }^{16}$ implies concerted efforts to effectively minimize the presence of excess body weight and pain among people with painful disabling osteoarthritis in a timely way is crucial. This brief describes some recent research concerning the link between obesity and painful osteoarthritis, and in light of the compliance challenges associated with dietary restriction, ${ }^{17}$ plus enormous length of time and resources required to do this effectively the writer proposes a more comprehensive and timely strategy for fostering weight reduction and minimizing functional declines attributable to the overall disease process among obese osteoarthritis sufferers based on the available research. Since the keystone of managing osteoarthritis is the ability to self manage the disease and/or reduce or minimize further disability in a timely way strategies that overlap with this approach are specifically highlighted

\section{Methods}

To obtain the desired information, the PubMed, Web of Science, Academic Search Premiere websites were searched using key terms: behavioural interventions, osteoarthritis, obesity, pain, self management, treatment. All English language related reports that addressed the study question retrieved between 2000-present times were accessed, read and analyzed for related content. All forms of research were deemed acceptable if they focused on the above mentioned themes. General articles on osteoarthritis were not included. All articles were reviewed for their relevance and are reported in narrative form, as this work is deemed exploratory. Results of a series of data collection efforts conducted previously are also briefly highlighted.

\section{Findings}

Studies on pain, body mass and function in osteoarthritis as reported by Miller et al., ${ }^{18}$ higher pain levels have been consistently observed among various cohorts of obese adults with knee osteoarthritis. Collins et al., ${ }^{19}$ who examined 1753 osteoarthritis cases 
over an extended period found obese cases had greater pain than non obese cases. This pain generally remained unchanged over a 6year period. However, when Rosales et al., ${ }^{20}$ examined the impact of pain relieving medication, and education, plus the adoption of a balanced diet and exercise at least 3 times a week among 38 osteoarthritis cases, they not only found no significant body mass variation but those with higher initial body mass indices were less successful than those with lower body mass indices. As well pain did not improve significantly and those with high body mass indices did not improve as much as those with lower body mass indices. It was felt that the prevailing physical incapacity as a result of the pain may have been one reason why the body mass indices remained unchanged over the study period. Because improved function did not reduce pain in a consistent fashion however, the authors suggested that perhaps the pain symptoms were not adequately assessed or adequately treated as a result. Moreover despite the researcher's efforts to enable the patients to adhere to the recommended diet the population adhered only very sparsely. It is possible that excess pain not only impacts function and energy expenditure but leads to negative cognitive states such as depression, ${ }^{5}$ and consequently less ability to focus on recommended treatments, or deleterious changes in body composition and weight gain as a result of pain..$^{13}$ In their study Graver et al., ${ }^{21}$ which assessed differences in mobility performance, self efficacy, pain and physical activity as a function of weight status observed obese class II patients were outperformed by their counterparts on all measures. In addition, among the class III obese participants their pain rating was $178 \%$ higher than the normal weight subjects. The presence of pain is known to predict walking difficulty and performance of everyday life. According to the authors, regardless of whether physical inactivity and mobility precede weight gain and osteoarthritis or whether the onset of osteoarthritis precedes mobility challenges and declining physical activity leads to obesity the fact that pain symptoms were exacerbated and physical activity levels were reduced in those with higher weight status suggests more needs to be done to impact this interacting series of overlapping problems. In particular, pain can be associated with inflammation, ${ }^{22}$ and joint effusion and muscle dysfunction which alone can reduce physical ability and the ability to maintain a healthy weight. Obesity too is independently associated with inflammation, ${ }^{21}$ and although exercise and weight loss advocated for managing osteoarthritis are effective strategies for managing overweight problems Thomazeau et al., ${ }^{23}$ found obese patients suffered higher bouts of acute pain than non obese patients and more unrelieved chronic pain, suggesting this obese group would be more reluctant to move or carry out physical activity recommendations, ${ }^{10}$ and was hence less active than patients with healthy body weights as a whole. This is important because data reveal that obese osteoarthritis cases not only express having more pain than those who are non obese, but they also incur more severe joint damage than the non obese patient.24 Unfortunately this obese subgroup commonly experiences excess discomfort during exercise do not perceive exercise to be enjoyable, and consider being sedentary the norm. ${ }^{10}$ As well those who are morbidly obese are found to have high pain catastrophizing beliefs, beliefs associated with binge eating behaviours. ${ }^{10}$ In turn those with more pain may be more likely to experience weight gain, ${ }^{10}$ and even small amounts of weight gain have adverse clinical implications ${ }^{25}$ such as perpetuating more mechanical disturbances as well as inflammatory reactions. ${ }^{10}$ Extrapolated from an earlier study series, ${ }^{26}$ (Figure 1) shows the distribution of body mass indices among a cohort of generally healthy community dwelling adults with mild to moderate knee osteoarthritis assessed in our laboratory. Among the 100 cases studied, mean age 69.7, (range 40-89), no subjects were identified as being underweight.
As outlined, those under age 60 , where osteoarthritis is not expected to be associated with this age group had higher body mass indices depression, and pain scores on average. Those with two affected joints $(\mathrm{N}=43)$ were slightly heavier on average than those with one affected joint $(\mathrm{N}=57)(\mathrm{BMI}=30$ and 28 respectively), suggesting a dose response trend that would best be prevented, rather than treated after the fact. Also noteworthy was that 25/42 obese cases exhibited depressive symptoms versus 17/42 that showed no signs of depression. As observed using Pearson correlations, those with higher body mass indices experienced more subjective pain $(\mathrm{r}=.27, \mathrm{p}=.003)$, as did those with higher body weights $(\mathrm{r}=.24 ; \mathrm{p}=.02)$ and as outlined in (Figure 2), suggest high body mass indices are likely to increase pain, regardless of age, and gender and it is possible that pain impedes the ability to lose weight, or fosters excess weight inadvertently through multiple mechanisms. ${ }^{10}$

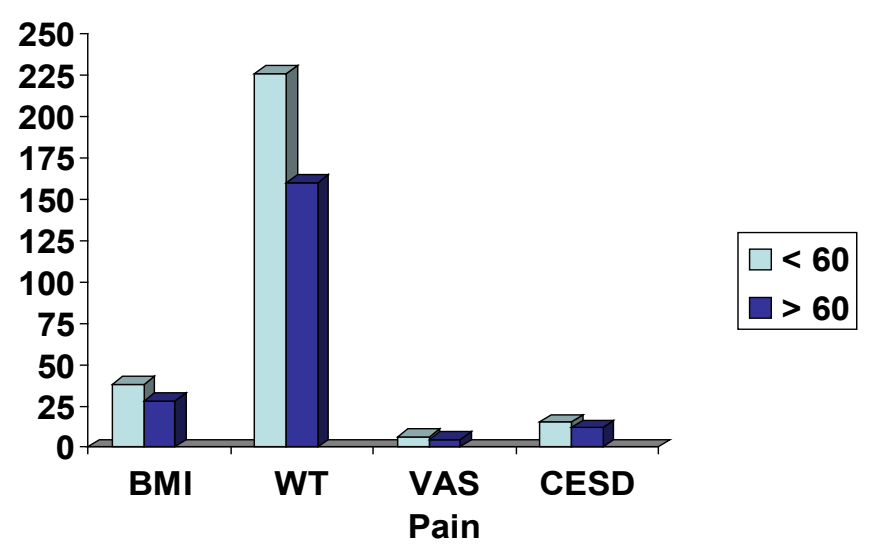

Figure I Comparison of body mass and pain and depression among cases younger than 60years, and older than 60years.

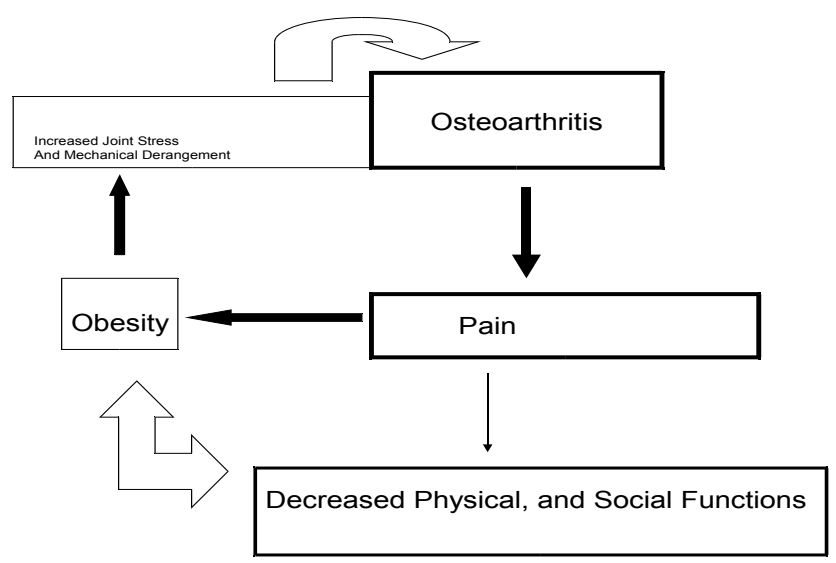

Figure 2 Schematic of Potential Explanation of Relationship between Obesity and Osteoarthritis.

Strategies to offset obesity among osteoarthritis cases among the strategies believed to allay the impact of excess body weight on the osteoarthritis process a key recommendation highly visible in the related literature is for people with osteoarthritis who are obese to undertake some form of weight loss. Although largely focused on dietary intake modifications ${ }^{27}$ or bariatric surgery ${ }^{28}$ in severe cases of obesity less emphasis has been placed on determining all the possible pathways through which obesity can adversely impact osteoarthritis incidence and progression and on developing parallel strategies to offset these differential and interacting factors. Efforts to provide 
standard restrictive diets and behavioural therapy directed at weight management while reducing body weight in controlled trials may not be optimally efficacious in the real world in the absence of parallel strategies to minimize the related inflammatory effects associated with obesity ${ }^{29}$ plus observations of deficient nutrient intake and poor eating behaviours among obese adults with chronic pain whose caloric intake was not excessive. ${ }^{30}$ In addition the importance of cognitive behavioural strategies directed towards countering excess stress pain catastrophizing, low self-efficacy, self-efficacy, and fear, all possible weight gain determinants, is strongly indicated, ${ }^{10}$ as are efforts to minimize joint pain through the application of non pharmacologic strategies, including strength training of the muscles around the affected joint to enhance a more favourable fat $/$ muscle ratio,${ }^{31}$ while enabling safe levels of increased physical activity levels. ${ }^{10,23}$ Since having a high body mass index predicts higher pain and disability levels over time, ${ }^{32}$ plus the accumulation of components of the metabolic syndrome which are significantly associated with a higher intensity of pain independent of weight, ${ }^{33}$ efforts to control pain that can lead to heightened activity practices may be especially advantageous for reducing excess muscle fat mass and inflammation that predisposes to abrasive joint damage. ${ }^{11}$ The development of disabling osteoarthritis, which can spread from one joint to multiple joints more rapidly in obese than non obese cases may be favourably impacted as well, ${ }^{10}$ as may surgical outcomes. ${ }^{15}$ More carefully construed multi-pronged comprehensive efforts if adopted as a standard of practice would not only have the potential to foster more rapid weight reduction and more effective weight maintenance after that but could potentially minimize the possible onset of insulin resistance that may adversely affect cartilage homeostasis,${ }^{34}$ plus the acquisition of other co morbid conditions such as hypertension, along with depression over time. In addition, educational efforts directed towards providing obese osteoarthritis patients with a better understanding of how some of their pain is likely to be produced by chemicals in fatty tissue, ${ }^{35}$ as well, providing an understanding of how obesity can increase the risk for heart disease, diabetes, and hypertension or worsen these pre existing conditions as well as pain would seem helpful. Explaining that joint replacement surgery designed to reduce pain and disability is often complicated by the presence of obesity, and that those with lower body weights suffer less pain than those with higher body weights may be useful as well. ${ }^{1,12,36}$ That is in addition to structured weight loss directives, preventative attempts to control pain plus the provision of thoughtful educational efforts that places emphasis on the benefits of weight loss including the lowered risk of excess osteoarthritis disability, and inflammatory body responses due to fatty tissue may prove highly successful. As well efforts to minimize stress may enable more optimal energy balance and activity levels, and may also reduce the risk of excess truncal obesity due to high cortisol levels, ${ }^{10}$ plus the risk of depression that can foster weight gain. ${ }^{6}$ As well, stressing the benefits of strength training for reducing fat deposits in muscle as well as for pain relief may be helpful because obesity makes it harder for the bone to absorb impact loads that can produce pain and joint dysfunction. ${ }^{37}$

To assist in this process, a team of physical therapists, counselors, dieticians, physicians, psychologists, cardiologists, and occupational therapists trained to carefully assess the individual patient in a holistic manner, and to provide a tailored intervention package for them is desirable. Baseline questionnaires and physical and mental health examinations, plus records of daily dietary intake and pain levels as well as follow up evaluations can be helpful as well. A focus on attaining a healthy weight, avoiding harmful joint damaging activities and on the provision of optimal cognitive behavioural strategies, stress and eating control strategies, while helping clients to meet daily nutrient and physical activity recommendations is especially recommended.

In sum, since those osteoarthritis cases with high body weights are likely to experience lower pain reduction levels when treated, ${ }^{38}$ and Cimmino et al., ${ }^{39}$ noted a significant relationship between pain and body mass $>$ or $=30(\mathrm{OR}=1.52 ; 95 \%$ CI 1.42 to 1.61$)$ among people with different forms of osteoarthritis, efforts to minimize pain among those people with osteoarthritis who are overweight is clearly indicated. However, even though weight reduction alone can ameliorate osteoarthritis symptoms, a high number of cases, may remain obese, with unchanged levels of pain over time. In addition, those who resort to narcotic or excess over the counter pharmaceutical drugs to relieve their pain are likely to have increased, rather than decreased weight problems. ${ }^{10}$ Depression accompanying the presence of pain and disability can heighten binge eating behaviours, as well as foster excess overweight due to anti-depressants medication usage. At the same time, lower levels of social and physical activities can impede energy balance strategies. Chronic pain may also alter glucose homeostasis negatively, fostering the potential loss of muscle mass, and the co-occurrence of fat gain. ${ }^{40}$ Sleep disturbances that arise as a consequence of pain, are also found to heighten the proclivity to weight gain, inadvertently, ${ }^{41}$ and the risk of having metabolic syndrome that may increase pain and joint damage. ${ }^{34}$ Thus rather than advocating for simple weight reduction to reduce osteoarthritis symptoms, which does not necessarily reduce body fat foster muscle strength gains, nor ameliorate joint malalignment problems and others such as depression, a specific focus on alleviating pain and those correlates that could have a bearing on body fat reduction as a therapeutic target, should not be underestimated as having direct as well as indirect benefits on both weight associated issues and overall disability. ${ }^{42}$ The importance of the need to limit excess weight gain at an early age as this heightens the risk for the onset of osteoarthritis later on, preventing weight gain in adults of healthy weight diagnosed with osteoarthritis, and reducing class III obesity levels to class II or I is strongly advocated as well. ${ }^{18}$ Moreover, among this patient group, minimizing the risk of other chronic conditions related to obesity, such as heart disease, hypertension, and diabetes, as well as stress and anxiety should be emphasized by all practitioners as all these conditions and adverse health implications can potentially impact the pathogenesis of both osteoarthritis as well as obesity, quite markedly and negatively. ${ }^{43}$ Advocating for healthy eating practices and ensuring vitamin D levels are optimal, and treating pain as a separate illness in parallel with other strategies ${ }^{7}$ may impact both pain, ${ }^{44}$ as well as obesity levels quite favourably.

\section{Conclusion}

A large body of data reveals that the natural course of osteoarthritis, including adverse surgical outcomes may be mediated or moderated or predated by the presence of an excess body mass. ${ }^{45,46}$ This may reflect a higher presence of body fat which is associated with more mechanical stress and derangement, ${ }^{47}$ as well as hormonal products that cause pain or act as inflammatory mediators that hasten cartilage degradation. ${ }^{48} \mathrm{It}$ is also associated with a greater infiltration of the muscles of the body by fat,${ }^{49}$ thus reducing muscle strength generating capacity and quality both generally and specifically, ${ }^{50}$ and thereby exposing the joint to greater impact than if the muscle bulk is well preserved, as well as widespread pain. ${ }^{31}$ As outlined in (Figure 2) there are consequently numerous ways for excess body weight to amplify joint damage directly or indirectly, through multiple pathways and since joint pain 
is the cardinal clinical complaint of people with osteoarthritis that may inhibit weight loss efforts attempts to unravel the source of both pain and obesity are likely to prove more fruitful than efforts conducted in isolation. However although most of the literature focuses on how obesity influences osteoarthritis pain, very little attention is devoted to the possibility that the presence of joint damage resulting in excess pain and immobility can foster weight gain and set off a cycle of pain and weight gain that is reinforcing as outlined by Jhanke et al., ${ }^{51}$ can produce dysfunctions of sleep, mood and energy that can adversely influence weight maintenance or reduction efforts. ${ }^{7}$ Also noteworthy in this context, is the fact that very little is written about the possibility of acquiring more extensive osteoarthritis at other joint sites in those with a single affected joint if they remain overweight as suggested by Marks, 26 which suggests timely weight reduction is imperative. The finding that the contribution of overweight to the pain experience in knee osteoarthritis may be more Aversive for females than males with the same diagnosis also suggests more should be done to explore the obesity pain link in this sub group.

In particular since being obese increases pain a significant predictor of greater functional decline, ${ }^{52-54}$ and stronger pain medications requirements, ${ }^{10}$ professionals interested in improving the outlook for people with osteoarthritis might want to consider the potential benefits of approaching this clinical condition by designing and implementing more comprehensive multi-pronged interventions in a timely way once a patient is diagnosed with this condition as recommended by Focht et al., ${ }^{55}$ Messier et al. ${ }^{56}$ and Peters et al.,${ }^{32}$ who found those osteoarthritis cases with more overall pain at baseline had more pain seven years later plus worse function and greater degrees of joint deterioration. Thomazeau et al. ${ }^{23}$ too found obese osteoarthritis cases suffered more significantly from chronic unrelieved pain than those with a healthy weight. As outlined by Powell et al., ${ }^{37}$ if obesity is the main preventable risk factor for the onset and progression of osteoarthritis, more concerted prevention efforts plus more impactful treatments to minimize obesity may vastly improve the quality of life and outlook for many across the globe,${ }^{57}$ along with allaying the immense suffering and social costs that presently persist and are predicted to increase. However as proposed by Janke et al., ${ }^{51}$ much more research focusing on expanding our knowledge about the relationship between pain and obesity testing explanatory models addressing their co-occurrence and developing efficacious treatments to effectively target this comorbidity is desirable given that longitudinal studies suggest overweight/obesity early in life is a risk factor for pain and is likely to be highly prevalent because of the increasing obesity proliferation. ${ }^{40}$ Moreover given the co-occurrence of pain and overweight/obesity and its negative effect on the management of obesity, ${ }^{58}$ and the importance of daily levels of optimal cyclic joint loading in joint preservation a well conceived pain reduction protocol would seem beneficial for reducing metabolic as well as mechanical determinants of osteoarthritis. Conversely a failure to control pain levels along with a lack of regular cyclic joint loading foster further inflammatory related joint destruction ${ }^{58}$ a low quality of life,$^{51}$ sleep disturbances which is a risk factor for obesity as well as depression and other important social economic and physical functions. In addition rates of hypertension diabetes and cardiovascular disease associated with greater rates of joint deterioration are likely to increase. ${ }^{57}$ Since weight loss alone may not alter the prevailing joint pathology, and may alter joint biomechanics adversely in some cases for example if there is excessive weight loss, and the cause of the problem is joint instability or malalignment and/or weight loss is accompanied by excess loss of lean muscle mass, a focus on this strategy alone may yet have highly negative implications for perpetuating osteoarthritis pain among those who are obese. Vitamin
D intake and other essential nutrients for health and joint function as well as pain perception44 may be especially negatively influenced in cases of bariatric surgery in the absence of careful nutrition education, due to persistent low levels of mobility and sunlight exposure in face of pain. Hence avoiding such extreme measures in efforts to reduce osteoarthritis pain in obese cases seems highly recommended. To this end, the use of whole body mass impedance measures and the ability to examine local and general body fat distribution versus lean muscle mass, which can contribute to a high body mass index value along with periodic vitamin D assays, followed by long term carefully designed tailored multi pronged interventions as outlined by Janke et al. ${ }^{59}$ and Barlow, ${ }^{60}$ as well as a focus on diet content, ${ }^{61}$ stress control, ${ }^{62}$ glucose control $^{63}$ and avoidance of reduced bone mineral density effects that can occur post weight loss ${ }^{64}$ is highly recommended in this respect, as is careful attention to maximizing mental health through cognitive behavioral therapy ${ }^{65,66}$ (Figure 3 ) and behavioral therapy combined, ${ }^{67,68}$ as well as overall health status, muscle and physical function through non pharmacologic approaches ${ }^{69,70}$ Finally ascertaining the cause of prevailing obesity and intervening accordingly in this respect is strongly advocated.

- Anxiety and fear

- Chronic stress

- Depression

- Excess unrelenting pain

- Food preparation challenges for hand osteoarthritis and lower leg and spine sufferers

- Lack of motivation

- Limited energy

- Low self-efficacy for dietary behaviors

- Low self esteem

- Low vitamin D levels

- Overuse of narcotic and anti-inflammatory agents

- Sleep disturbances

- Socioeconomic problems affecting food choices, long term care support

Figure 3 Potential Barriers to Weight Reduction in People with Disabling Painful Osteoarthritis that May be Overcome by a Carefully Designed Program of Appropriate Dietary Strategies, Pain Relief Strategies, Stress Control Strategies, Lifestyle Behavior and Cognitive Behavior Therapy Interventions. ${ }^{66}$

\section{Acknowledgements}

None.

\section{Conflict of interest}

The author declares no conflict of interest.

\section{References}

1. Jones G. Osteoarthritis: Where are we for pain and therapy in 2013? Aust Fam Physician. 2013;42(11):766-769.

2. Salaffi F, Ciapetti A, Carotti M. The sources of pain in osteoarthritis: a pathophysiological review. Reumatismo. 2014;66(1):57-71.

3. Cross M, Smith E, Hoy D, et al. The global burden of hip and knee osteoarthritis: estimates from the Global Burden of Disease 2010 study. Ann Rheum Dis. 2014;73(7):1323-1330.

4. Jhun HJ, Sung NJ, Kim SY. Knee pain and its severity in elderly Koreans: prevalence, risk factors and impact on quality of life. J Korean Med Sci. 2013;28(12):1807-1813. 
5. Veenhof C, Huisman PA, Barten JA, et al. Factors associated with physical activity in patients with osteoarthritis of the hip or knee: a systematic review. Osteoarthritis Cartilage. 2012;20(1):6-12.

6. Lin KP, Liang TL, Liao IC, et al. Associations among depression, obesity, and metabolic syndrome in young adult females. Biol Res Nurs. 2013;16(3):327-334

7. Fitzcharles MA, Shir Y. Management of chronic pain in the rheumatic diseases with insights for the clinician. Ther Adv Musculoskelet Dis. 2011;3(4):179-190.

8. Cheskin LJ, Bartlett SJ, Zayas R, et al. Prescription medications: a modifiable contributor to obesity. South Med J. 1999;92(9):898-904.

9. Ruetsch O, Viala A, Bardou H, et al. [Psychotropic drugs induced weight gain. a review of the literature concerning epidemiological data, mechanisms and management]. Encephale. 2005;31(4 Pt 1):507-516.

10. Vincent HK, Adams MC, Vincent KR, et al. Musculoskeletal pain fear avoidance behaviors, and functional decline in obesity: potentia interventions to manage pain and maintain function. Reg Anesth Pain Med. 2013;38(6):481-491.

11. Rodriguez RJ, Escobar-Linares LE, Garcia-Carrasco M, et al. The relationship between the metabolic syndrome and energy-utilization deficit in the pathogenesis of obesity-induced osteoarthritis. Med Hypotheses. 2007;69(4):860-868.

12. Bliddal H, Leeds AR, Christensen R. Osteoarthritis, obesity and weight loss: evidence, hypotheses and horizons - a scoping review. Obes Rev. 2014;15(7):578-586.

13. Cicuttini FM, Wluka AE. Osteoarthritis: Is OA a mechanical or systemic disease? Nat Rev Rheumatol. 2014;10(9):515-516.

14. Watts CD, Wagner ER, Houdek MT, et al. Morbid obesity: a significant risk factor for failure of two-stage revision total knee arthroplasty for infection. J Bone Joint Surg Am. 2014;96(18):e154.

15. Collins JA, Beutel BG, Garofolo G, et al. Correlation of obesity with patient-reported outcomes and complications after hip arthroscopy. Arthroscopy. 2015;31(1):57-62.

16. Alford DP. Weighing in on opioids for chronic pain: the barriers to change. JAMA. 2013;310(13):1351-1352.

17. Templeton CL, Petty BJ, Harter JL. Weight control - a group approach for arthritis clients. J Nutr Ed. 1978;10(1):33-35.

18. Miller GD, Rejeski WJ, Williamson JD, et al. The Arthritis, Diet and Activity Promotion Trial (ADAPT):design, rationale, and baseline results. Control Clin Trials. 2003;24(4):462-480.

19. Collins JE, Katz JN, Dervan EE, et al. Trajectories and risk profiles of pain in persons with radiographic, symptomatic knee osteoarthritis: data from the osteoarthritis initiative. Osteoarthritis Cartilage. 2014;22(5):622-630.

20. Rosales Ade L, Brito NL, Frucchi R, et al. Obesity, ostearthritis and clinical treatment. Acta Ortop Bras. 2014;22(3):136-139.

21. Garver MJ, Focht BC, Dials J, et al. Weight status and differences in mobility performance, pain symptoms, and physical activity in older, knee osteoarthritis patients. Arthritis. 2014;2014:375909.

22. Iannone F, Lapadula G. Obesity and inflammation-targets for OA therapy. Curr Drug Targets. 2010;11(5):586-598.

23. Thomazeau J, Perin J, Nizard R, et al. Pain management and pain characteristics in obese and normal weight patients before joint replacement. J Eval Clin Pract. 2014;5(20):611-616

24. Widhalm HK, Seemann R, Hamboeck M, et al. Osteoarthritis in morbidly obese children and adolescents, an age-matched controlled study. Knee Surg Sports Traumatol Arthrosc. 2016;24(3):644-652.
25. Teichtahl AJ, Wluka AE, Tanamas SK, et al. Weight change and change in tibial cartilage volume and symptoms in obese adults. Ann Rheum Dis. 2015;74(6):1024-1029.

26. Marks R. Obesity profiles with knee osteoarthritis: correlation with pain, disability, disease progression. Obesity (Silver Spring). 2007;15(7):18671874.

27. Abiles V, Rodriguez-Ruiz S, Abiles J, et al. Effectiveness of cognitivebehavioral therapy in morbidity obese candidates for bariatric surgery with and without binge eating disorder. Nutr Hosp. 2013;28(5):15231529

28. Annesi JJ, Tennant GA. From morbid obesity to a healthy weight using cognitive-behavioral methods: a woman's three-year process with one and one-half years of weight maintenance. Perm J. 2012;16(4):54-59.

29. King LK, March L, Anandacoomarasamy A. Obesity \& osteoarthritis. Indian J Med Res. 2013;138(2):185-193.

30. Meleger AL, Froude CK, Walker J. Nutrition and eating behavior in patients with chronic pain receiving long-term opioid therapy. $P M R$. 2014;6(1):7-12.

31. Yoo JJ, Lim SH, Cho NH, et al. Relationships between body mass index, fat mass, muscle mass, and musculoskeletal pain in community residents. Arthritis Rheumatol. 2014;66(12):3511-3520.

32. Peters TJ, Sanders C, Dieppe P, et al. Factors associated with change in pain and disability over time: a community-based prospective observational study of hip and knee osteoarthritis. Br J Gen Pract. 2005;55(512):205-211.

33. Shin D (2014) Association between metabolic syndrome, radiographic knee osteoarthritis, and intensity of knee pain: results of a national survey. J Clin Endocrinol Metab 99(9):3177-3183.

34. Dubrolowski C. Is osteoarthritis part of a metabolic syndrome? Retrieved from Ezine articles. 2009.

35. Gandhi R, Perruccio AV, Rizek R, et al. Obesity-related adipokines predict patient-reported shoulder pain. Obes Facts. 2012;6(6):536-541

36. Cimmino MA, Scarpa R, Caporali R, et al. Body mass and osteoarthritis pain: results from a study in general practice. Clin Exp Rheumatol. 2013;31(6):843-849.

37. Powell A, Teichtahl AJ, Wluka AE, et al. Obesity: a preventable risk factor for large joint osteoarthritis which may act through biomechanical factors. Br J Sports Med. 2005;39(1):4-5.

38. Gudbergsen H, Boesen M, Lohmander LS, et al. Weight loss is effective for symptomatic relief in obese subjects with knee osteoarthritis independently of joint damage severity assessed by high-field MRI and radiography. Osteoarthritis Cartilage. 2012;20(6):495-502.

39. Cimmino MA, Sarzi-Puttini P, Scarpa R, et al. Clinical presentation of osteoarthritis in general practice: determinants of pain in Italian patients in the AMICA study. Semin Arthritis Rheum. 2005;35(1 Suppl 1):17-23.

40. Sowers MR, Karvonen Guterrez CA. The evolving role of obesity in knee osteoarthritis. Curr Opin Rheumatol. 2012;22(5):533-537.

41. Bayon V, Leger D, Gomez-Merino D, et al. Sleep debt and obesity. Ann med. 2014;46(5):264-272.

42. Toda Y, Toda T, Takemura S, et al. Change in body fat, but not body weight or metabolic correlates of obesity, is related to symptomatic relief of obese patients with knee osteoarthritis after a weight control program. J Rheumatol. 1998;25(11):2181-2186.

43. Pottie P, Presle N, Terlain B, et al. Obesity and osteoarthritis: more complex than predicted! Ann Rheum Dis. 2006;65(11):1403-1405.

44. von Kanel R, Muller-Hartmannsgruber V, Kokinogenis G, et al. Vitamin $\mathrm{d}$ and central hypersensitivity in patients with chronic pain. Pain Med. 2014;15(9):1609-1618 
45. Berenbaum F, Sellam J. Obesity and osteoarthritis: what are the links? Joint Bone Spine. 2008;75(6):667-668.

46. Paradowski PT, Englund M, Lohmander LS, et al. The effect of patient characteristics on variability in pain and function over two years in early knee osteoarthritis. Health Qual Life Outcomes. 2005;3:59.

47. Oyeyemi AL. Body mass index, pain and function in individuals with knee osteoarthritis. Niger Med J. 2013;54(4):230-235.

48. Griffen TM, Guilak F. The role of mechanical loading in the onset and progression of osteoarthritis. Exerc Sport Sci Rev. 2005;33(4):195-200.

49. Visser M, Kritchevssy SB, Goodpaster BH, et al. Longitudinal Aging Study Amsterdam. Leg muscle mass and composition in relation to lower extremity performance in men and women aged 70 to 79: the health, aging and body composition study. J Am Geriatr Soc. 2002;50(5):897904.

50. Newman AB, Haggerty CL, Goodpaster B, et al. Strength and muscle quality in a well-functioning cohort of older adults: the Health, Aging and Body Composition Study. J Am Geriatr Soc. 2003;51(3):323-330.

51. Janke EA, Collins A, Kozak AT. Overview of the relationship between pain and obesity: What do we know? Where do we go next? J Rehabil Res Dev. 2007;44(2):245-262.

52. Zhai G, Blizzard L, Srikanth V, et al. Correlates of knee pain in older adults: Tasmanian Older Adult Cohort Study. Arthritis Rheum15. 2006;55(2):264-271

53. Sinkov V, Cymet T. Osteoarthritis: understanding the pathophysiology, genetics, and treatments. J Natl Med Asso. 2003;95(6):475-482.

54. Issa SN, Sharma L. Epidemiology of osteoarthritis: an update. Curr Rheumatol Rep. 2006;8(1):7-15.

55. Focht BC, Rejeski WJ, Ambrosius WT, et al. Exercise, self-efficacy, and mobility performance in overweight and obese older adults with knee osteoarthritis. Arthritis Rheum. 2005;53(5):659-665.

56. Messier SP, Gutekunst DJ, Davis C, et al. Weight loss reduces kneejoint loads in overweight and obese older adults with knee osteoarthritis. Arthritis Rheum. 2005;2(7):2026-2032.

57. Okoro CA, Hootman JM, Strine TW, et al. Disability, arthritis, and body weight among adults 45 years and older. Obesity Res. 2004;12(5):854 861

58. Issa RI, Griffin TM. Pathobiology of obesity and osteoarthritis: integrating biomechanics and inflammation. Pathobiol Aging Age Relat Dis. 2012;9(2012):17470.

59. Janke EA, Fritz M, Hopkins C, et al. A randomized clinical trial of an integrated behavioral self-management intervention Simultaneously Targeting Obesity and Pain: the STOP trial. BMC Public Health 2014;14:621.
60. Barlow JH, Turner AP, Wright CC. A randomized controlled study of the Arthritis Self-Management Programmed in the UK. Health Educe Res. 2000;15(6):665-680.

61. Christensen P, Bartels EM, Riecke BF, et al. Improved nutritional status and bone health after diet-induced weight loss in sedentary osteoarthritis patients: a prospective cohort study. Eur J Clin Nutr. 2012;66(4):504 509.

62. Dallman MF, Pecoraro NC, la Fleur SE. Chronic stress and comfort foods: self-medication and abdominal obesity. Brain Behave Immune. 2005;19(4):275-280

63. Rasheed Z, Akhtar N, Haqqi TM. Advanced glycation end products induce the expression of interleukin -6 and interleukin- 8 by receptor for advanced glycation end product-mediated activation of mutagenactivated protein kinesis and nuclear factor $-\kappa \mathrm{B}$ in human osteoarthritis chondrocytes. Rheumatology (Oxford). 2011;50(5):838-851.

64. Beavers DP, Beavers KM, Loeser RF, et al. The independent and combined effects of intensive weight loss and exercise training on bone mineral density in overweight and obese older adults with osteoarthritis. Osteoarthritis Cartilage. 2014;22(6):726-733.

65. Gade H, Hjelmesæth J, Rosenvinge JH, et al. Effectiveness of a cognitive behavioral therapy for dysfunctional eating among patients admitted for bariatric surgery: a randomized controlled trial. J Obes 2014;2014:127936

66. Rapport L, Clark M, Wardle J. Evaluation of a modified cognitivebehavioural programme for weight management. Into $J$ Obes Relat Metab Disord. 2000;24(12):1726-1737.

67. Fabricator AN. Behavior therapy and cognitive-behavioral therapy of obesity: is there a difference? J Am Diet Assoc. 2007;107(1):92-99.

68. Brennan L, Walkley J, Wilks R, et al. Physiological and behavioral outcomes of a randomized controlled trial of a cognitive behavioral lifestyle intervention for overweight and obese adolescents. Obes Res Clin Pract. 2013;7(1):e23-e41.

69. Vitiello MV, McCurry SM, Shortreed SM, et al. Cognitive-behavioral treatment for co morbid insomnia and osteoarthritis pain in primary care: the lifestyles randomized controlled trial. J Am Geriatric Soc. 2013;61(6):947-956

70. Helminen EE, Sinikallio SH, Valjakka AL, et al. Effectiveness of cognitive-behavioral group intervention for knee osteoarthritis pain: protocol of a randomized controlled trial. BMC Musculoskeletal Disord. 2013;14:46. 